\title{
Discordant Antibiotic Therapy and Length of Stay in Children Hospitalized for Urinary Tract Infection
}

\author{
Karen E. Jerardi, MD ${ }^{1 \star}$, Katherine A. Auger, MD², Samir S. Shah, MD, MSCE ${ }^{1,3}$, Matthew Hall, PhD ${ }^{4}$, Paul D. Hain, MD5, \\ Angela L. Myers, MD, MPH${ }^{6}$, Derek J. Williams, MD, MPH${ }^{5}$, Joel S. Tieder, MD, $\mathrm{MPH}^{7}$
}

\begin{abstract}
${ }^{1}$ Division of Hospital Medicine, Cincinnati Children's Hospital Medical Center, Cincinnati, Ohio; 2Robert Wood Johnson Clinical Scholars Fellow, Department of Pediatrics and Communicable Diseases, University of Michigan, Ann Arbor, Michigan; ${ }^{3}$ Division of Infectious Diseases, Cincinnati Children's Hospital Medical Center, Cincinnati, Ohio; ${ }^{4}$ Children's Hospital Association, Shawnee Mission, Kansas; ${ }^{5}$ Division of Hospital Medicine, University of Texas Southwestern Medical Center, Dallas, Texas; ' ${ }^{S}$ ection of Infectious Diseases, Children's Mercy Hospital and Clinics, University of Missouri-Kansas City, Kansas City, Missouri; ' $D$ Department of Pediatrics, University of Washington School of Medicine and the Division of Hospital Medicine, Seattle Children's Hospital, Seattle, Washington.
\end{abstract}

BACKGROUND: Urinary tract infections (UTIs) are a common reason for pediatric hospitalizations.

OBJECTIVE: To determine the effect of discordant antibiotic therapy (in vitro nonsusceptibility of the uropathogen to initial antibiotic) on clinical outcomes for children hospitalized for UTI.

DESIGN/SETTING: Multicenter retrospective cohort study in children aged 3 days to 18 years, hospitalized at 5 children's hospitals with a laboratoryconfirmed UTI. Data were obtained from medical records and the Pediatric Hospital Information System (PHIS) database.

PARTICIPANTS: Patients with laboratory-confirmed UTI.

MAIN EXPOSURE: Discordant antibiotic therapy.

MEASUREMENTS: Length of stay and fever duration. Covariates included age, sex, insurance, race, vesicoureteral reflux, antibiotic prophylaxis, genitourinary abnormality, and chronic care conditions.

RESULTS: The median age of the 216 patients was 2.46 years (interquartile range [IQR]: $0.27,8.89$ ) and $25 \%$ were male. The most common causative organisms were $E$. coli and Klebsiella species. Discordant therapy occurred in 10\% of cases and most commonly in cultures positive for Klebsiella species, Enterobacter species, and mixed organisms. In adjusted analyses, discordant therapy was associated with a 1.8 day $(95 \%$ confidence interval $[\mathrm{Cl}]: 1.5,2.1)$ longer length of stay [LOS], but not with fever duration.

CONCLUSIONS: Discordant antibiotic therapy for UTI is common and associated with longer hospitalizations. Further research is needed to understand the clinical factors contributing to the increased LOS and to inform decisions for empiric antibiotic selection in children with UTIs. Journal of Hospital Medicine 2012;7:622-627. (c) 2012 Society of Hospital Medicine
Urinary tract infections (UTIs) are one of the most common reasons for pediatric hospitalizations. ${ }^{1}$ Bacterial infections require prompt treatment with appropriate antimicrobial agents. Results from culture and susceptibility testing, however, are often unavailable until 48 hours after initial presentation. Therefore, the clinician must select antimicrobials empirically, basing decisions on likely pathogens and local resistance patterns. ${ }^{2}$ This decision is challenging because the effect of treatment delay on clinical outcomes is difficult to determine and resistance among uropathogens is increasing. Resistance rates have doubled over the past several years. ${ }^{3,4}$ For common first-line antibiotics, such as ampicillin and trimethoprim-sulfamethoxazole,

*Address for correspondence and reprint requests: Karen E. Jerardi, MD, 3333 Burnet Ave, 5th Floor, Kasota Bldg, MLC 9016, Cincinnati, OH 45229-3039; Telephone: 513-636-4506; Fax: 513-636-7247; E-mail: Karen.Jerardi@cchmc.org

Additional Supporting Information may be found in the online version of this article.

Received: March 27, 2012; Revised: May 31, 2012; Accepted: June 15 2012

2012 Society of Hospital Medicine DOI 10.1002/jhm.1960

Published online in Wiley Online Library (Wileyonlinelibrary.com). resistance rates for Escherichia coli, the most common uropathogen, exceed $25 \% .^{4,5}$ While resistance to third-generation cephalosporins remains low, rates in the United States have increased from $<1 \%$ in 1999 to $4 \%$ in 2010 . International data shows much higher resistance rates for cephalosporins in general. ${ }^{6,7}$ This high prevalence of resistance may prompt the use of broad-spectrum antibiotics for patients with UTI. For example, the use of third-generation cephalosporins for UTI has doubled in recent years. ${ }^{3}$ Untreated, UTIs can lead to serious illness, but the consequences of inadequate initial antibiotic coverage are unknown., ${ }^{8,9}$

Discordant antibiotic therapy, initial antibiotic therapy to which the causative bacterium is not susceptible, occurs in up to $9 \%$ of children hospitalized for UTI. ${ }^{10}$ However, there is reason to believe that discordant therapy may matter less for UTIs than for infections at other sites. First, in adults hospitalized with UTIs, discordant initial therapy did not affect the time to resolution of symptoms. ${ }^{11,12}$ Second, most antibiotics used to treat UTIs are renally excreted and, thus, antibiotic concentrations at the site of infection are higher than can be achieved in the serum or cerebrospinal fluid. ${ }^{13}$ The Clinical and Laboratory 
Standard Institute has acknowledged that traditional susceptibility breakpoints may be too conservative for some non-central nervous system infections; such as non-central nervous system infections caused by Streptococcus pneumoniae. ${ }^{14}$

As resistance rates increase, more patients are likely to be treated with discordant therapy. Therefore, we sought to identify the clinical consequences of discordant antimicrobial therapy for patients hospitalized with a UTI.

\section{METHODS}

\section{Design and Setting}

We conducted a multicenter, retrospective cohort study. Data for this study were originally collected for a study that determined the accuracy of individual and combined International Classification of Diseases, Ninth Revision (ICD-9) discharge diagnosis codes for children with laboratory tests for a UTI, in order to develop national quality measures for children hospitalized with UTIs. ${ }^{15}$ The institutional review board for each hospital (Seattle Children's Hospital, Seattle, WA; Monroe Carell Jr Children's Hospital at Vanderbilt, Nashville, TN; Cincinnati Children's Hospital Medical Center, Cincinnati, OH; Children's Mercy Hospital, Kansas City, MO; Children's Hospital of Philadelphia, Philadelphia, PA) approved the study.

\section{Data Sources}

Data were obtained from the Pediatric Health Information System (PHIS) and medical records for patients at the 5 participating hospitals. PHIS contains clinical and billing data from hospitalized children at 43 freestanding children's hospitals. Data quality and coding reliability are assured through a joint effort between the Children's Hospital Association (Shawnee Mission, KS) and participating hospitals. ${ }^{16}$ PHIS was used to identify participants based on presence of discharge diagnosis code and laboratory tests indicating possible UTI, patient demographics, antibiotic administration date, and utilization of hospital resources (length of stay [LOS], laboratory testing).

Medical records for each participant were reviewed to obtain laboratory and clinical information such as past medical history (including vesicoureteral reflux [VUR], abnormal genitourinary [GU] anatomy, use of prophylactic antibiotic), culture data, and fever data. Data were entered into a secured centrally housed web-based data collection system. To assure consistency of chart review, all investigators responsible for data collection underwent training. In addition, 2 pilot medical record reviews were performed, followed by group discussion, to reach consensus on questions, preselected answers, interpretation of medical record data, and parameters for free text data entry.

\section{Subjects}

The initial cohort included 460 hospitalized patients, aged 3 days to 18 years of age, discharged from participating hospitals between July 1, 2008 and June 30, 2009 with a positive urine culture at any time during hospitalization. ${ }^{15}$ We excluded patients under 3 days of age because patients this young are more likely to have been transferred from the birthing hospital for a complication related to birth or a congenital anomaly. For this secondary analysis of patients from a prior study, our target population included patients admitted for management of UTI. ${ }^{15}$ We excluded patients with a negative initial urine culture $(\mathrm{n}=59)$ or if their initial urine culture did not meet definition of laboratory-confirmed UTI, defined as urine culture with $>50,000$ colony-forming units (CFU) with an abnormal urinalysis (UA) $(\mathrm{n}=77)^{1,17-19}$ An abnormal UA was defined by presence of white blood cells, leukocyte esterase, bacteria, and/or nitrites. For our cohort, all cultures with $>50,000 \mathrm{CFU}$ also had an abnormal urinalysis. We excluded 19 patients with cultures classified as 10,000-100,000 CFU because we could not confirm that the CFU was $>50,000$. We excluded 30 patients with urine cultures classified as "normal" or "mixed" flora, positive for a mixture of organisms not further identified, or if results were unavailable. Additionally, coagulase-negative Staphylococcus species $(\mathrm{n}=8)$ were excluded, as these are typically considered contaminants in the setting of urine cultures. ${ }^{2}$ Patients likely to have received antibiotics prior to admission, or develop a UTI after admission, were identified and removed from the cohort if they had a urine culture performed more than 1 day before, or 2 days after, admission $(\mathrm{n}=$ $35)$. Cultures without resistance testing to the initial antibiotic selection were also excluded $(\mathrm{n}=16)$.

\section{Main Outcome Measures}

The primary outcome measure was hospital LOS. Time to fever resolution was a secondary outcome measure. Fever was defined as temperature $\geq 38^{\circ} \mathrm{C}$. Fever duration was defined as number of hours until resolution of fever; only patients with fever at admission were included in this subanalysis.

\section{Main Exposure}

The main exposure was initial antibiotic therapy. Patients were classified into 3 groups according to initial antibiotic selection: those receiving 1) concordant; 2) discordant; or 3) delayed initial therapy. Concordance was defined as in vitro susceptibility to the initial antibiotic or class of antibiotic. If the uropathogen was sensitive to a narrow-spectrum antibiotic (eg, first-generation cephalosporin), but was not tested against a more broad-spectrum antibiotic of the same class (eg, third-generation cephalosporin), concordance was based on the sensitivity to the narrow-spectrum antibiotic. If the uropathogen was sensitive to a 
broad-spectrum antibiotic (eg, third-generation cephalosporin), concordance to a more narrow-spectrum antibiotic was not assumed. Discordance was defined as laboratory confirmation of in vitro resistance, or intermediate sensitivity of the pathogen to the initial antibiotic or class of antibiotics. Patients were considered to have a delay in antibiotic therapy if they did not receive antibiotics on the day of, or day after, collection of UA and culture. Patients with more than 1 uropathogen identified in a single culture were classified as discordant if any of the organisms was discordant to the initial antibiotic; they were classified as concordant if all organisms were concordant to the initial antibiotic. Antibiotic susceptibility was not tested in some cases $(\mathrm{n}=16)$.

Initial antibiotic was defined as the antibiotic(s) billed on the same day or day after the UA was billed. If the patient had the UA completed on the day prior to admission, we used the antibiotic administered on the day of admission as the initial antibiotic.

\section{Covariates}

Covariates were selected a priori to include patient characteristics likely to affect patient outcomes; all were included in the final analysis. These were age, race, sex, insurance, disposition, prophylactic antibiotic use for any reason (VUR, oncologic process, etc), presence of a chronic care condition, and presence of VUR or GU anatomic abnormality. Age, race, sex, and insurance were obtained from PHIS. Medical record review was used to determine prophylactic antibiotic use, and presence of VUR or GU abnormalities (eg, posterior urethral valves). Chronic care conditions were defined using a previously reported method. ${ }^{20}$

\section{Data Analysis}

Continuous variables were described using median and interquartile range (IQR). Categorical variables were described using frequencies. Multivariable analyses were used to determine the independent association of discordant antibiotic therapy and the outcomes of interest. Poisson regression was used to fit the skewed LOS distribution. The effect of antibiotic concordance or discordance on LOS was determined for all patients in our sample, as well as for those with a urine culture positive for a single identified organism. We used the Kruskal-Wallis test statistic to determine the association between duration of fever and discordant antibiotic therapy, given that duration of fever is a continuous variable. Generalized estimating equations accounted for clustering by hospital and the variability that exists between hospitals.

\section{RESULTS}

Of the initial 460 cases with positive urine culture growth at any time during admission, 216 met inclusion criteria for a laboratory-confirmed UTI from urine culture completed at admission. The median age

\begin{tabular}{|c|c|c|c|c|c|}
\hline & Overall & Concordant $^{*}$ & Discordant $^{\dagger}$ & $\begin{array}{l}\text { Delayed } \\
\text { Antibiotics }^{\ddagger}\end{array}$ & $\begin{array}{l}P \\
\text { Value }\end{array}$ \\
\hline N & 216 & $178(82.4)$ & $22(10.2)$ & $16(7.4)$ & \\
\hline \multicolumn{6}{|l|}{ Gender } \\
\hline Male & $54(25.0)$ & $40(22.5)$ & $8(36.4)$ & $6(37.5)$ & \multirow[t]{2}{*}{0.18} \\
\hline Female & $162(75.0)$ & $138(77.5)$ & $14(63.64)$ & $10(62.5)$ & \\
\hline \multicolumn{6}{|l|}{ Race } \\
\hline Non-Hispanic white & $136(63.9)$ & $110(62.5)$ & $15(71.4)$ & $11(68.8)$ & \multirow[t]{5}{*}{0.83} \\
\hline Non-Hispanic black & $28(13.2)$ & $24(13.6)$ & $2(9.5)$ & $2(12.5)$ & \\
\hline Hispanic & $20(9.4)$ & $16(9.1)$ & $3(14.3)$ & $1(6.3)$ & \\
\hline Asian & $10(4.7)$ & $9(5.1)$ & $1(4.7)$ & & \\
\hline Other & $19(8.9)$ & $17(9.7)$ & & $2(12.5)$ & \\
\hline \multicolumn{6}{|l|}{ Payor } \\
\hline Government & $97(44.9)$ & $80(44.9)$ & $11(50.0)$ & $6(37.5)$ & \multirow[t]{3}{*}{0.58} \\
\hline Private & $70(32.4)$ & $56(31.5)$ & $6(27.3)$ & $8(50.0)$ & \\
\hline Other & $49(22.7)$ & $42(23.6)$ & $5(22.7)$ & $2(12.5)$ & \\
\hline \multicolumn{6}{|l|}{ Disposition } \\
\hline Home & $204(94.4)$ & $168(94.4)$ & $21(95.5)$ & $15(93.8)$ & \multirow[t]{3}{*}{0.99} \\
\hline Died & $1(0.5)$ & $1(0.6)$ & & & \\
\hline Other & $11(5.1)$ & $9(5.1)$ & $1(4.6)$ & $1(6.3)$ & \\
\hline \multicolumn{6}{|l|}{ Age } \\
\hline $3 d-60 d$ & $40(18.5)$ & 35 (19.7) & $3(13.6)$ & $2(12.5)$ & \multirow[t]{4}{*}{0.53} \\
\hline $61 \mathrm{~d}-2 \mathrm{y}$ & $62(28.7)$ & $54(30.3)$ & $4(18.2)$ & $4(25.0)$ & \\
\hline $3 y-12 y$ & $75(34.7)$ & $61(34.3)$ & $8(36.4)$ & $6(37.5)$ & \\
\hline $13 y-18 y$ & $39(18.1)$ & $28(15.7)$ & $7(31.8)$ & $4(25.0)$ & \\
\hline \multicolumn{6}{|l|}{ Length of stay } \\
\hline $1 d-5 d$ & $171(79.2)$ & $147(82.6)$ & $12(54.6)$ & $12(75.0)$ & \multirow[t]{4}{*}{0.03} \\
\hline $6 d-10 d$ & $24(11.1)$ & $17(9.6)$ & $5(22.7)$ & $2(12.5)$ & \\
\hline $11 d-15 d$ & $10(4.6)$ & $5(2.8)$ & $3(13.6)$ & $2(12.5)$ & \\
\hline $16 \mathrm{~d}+$ & $11(5.1)$ & $9(5.1)$ & $2(9.1)$ & 0 & \\
\hline \multicolumn{6}{|c|}{ Complex chronic conditions } \\
\hline Any CCC & $94(43.5)$ & $77(43.3)$ & $12(54.6)$ & $5(31.3)$ & 0.35 \\
\hline Cardiovascular & $20(9.3)$ & $19(10.7)$ & & $1(6.3)$ & 0.24 \\
\hline Neuromuscular & $34(15.7)$ & $26(14.6)$ & $7(31.8)$ & $1(6.3)$ & 0.06 \\
\hline Respiratory & $6(2.8)$ & $6(3.4)$ & & & 0.52 \\
\hline Renal & $26(12.0)$ & $21(11.8)$ & $4(18.2)$ & $1(6.3)$ & 0.52 \\
\hline Gastrointestinal & $3(1.4)$ & $3(1.7)$ & & & 0.72 \\
\hline $\begin{array}{l}\text { Hematologic/ } \\
\text { immunologic }\end{array}$ & $1(0.5)$ & & $1(4.6)$ & & 0.01 \\
\hline Metabolic & $8(3.7)$ & $6(3.4)$ & $1(4.6)$ & $1(6.3)$ & 0.82 \\
\hline $\begin{array}{l}\text { Congenital or } \\
\text { genetic }\end{array}$ & $15(6.9)$ & $11(6.2)$ & $3(13.6)$ & $1(6.3)$ & 0.43 \\
\hline Malignancy & $5(2.3)$ & $3(1.7)$ & $2(9.1)$ & & 0.08 \\
\hline VUR & $28(13.0)$ & $23(12.9)$ & $3(13.6)$ & $2(12.5)$ & 0.99 \\
\hline Abnormal GU & $36(16.7)$ & 31 (17.4) & $4(18.2)$ & $1(6.3)$ & 0.51 \\
\hline Prophylactic antibiotics & $67(31.0)$ & $53(29.8)$ & $10(45.5)$ & $4(25.0)$ & 0.28 \\
\hline
\end{tabular}

NOTE: Values listed as number (percentage). Abbreviations: CCC, complex chronic condition; GU, genitourinary; VUR, vesicoureteral reflux. ${ }^{*}$ In vitro susceptibility of uropathogen to initial antibiotic. ${ }^{\dagger}$ In vitro nonsusceptibility of uropathogen to initial antibiotic. ${ }^{\ddagger}$ No antibiotics given on day of, or day after, urine culture collection.

was 2.46 years (IQR: $0.27,8.89$ ). In the study population, $25.0 \%$ were male, $31.0 \%$ were receiving prophylactic antibiotics, $13.0 \%$ had any grade of VUR, and $16.7 \%$ had abnormal GU anatomy (Table 1 ). A total of $82.4 \%$ of patients were treated with concordant initial therapy, $10.2 \%$ with discordant initial therapy, and $7.4 \%$ received delayed initial antibiotic therapy. There were no significant differences between the groups for any of the covariates. Discordant antibiotic cases ranged from $4.9 \%$ to $21.7 \%$ across hospitals.

The most common causative organisms were E. coli $(65.7 \%)$ and Klebsiella spp $(9.7 \%)$ (Table 2). The 
TABLE 2. UTIS by Primary Culture Causative Organism

\begin{tabular}{lccll}
\hline Organism & Cases & $\begin{array}{l}\text { Concordant } \\
\text { No. }(\%)\end{array}$ & $\begin{array}{l}\text { Discordant }^{\dagger} \\
\text { No. (\%) }\end{array}$ & $\begin{array}{l}\text { Delayed } \\
\text { Antibiotics }^{\ddagger} \\
\text { No. (\%) }\end{array}$ \\
\hline E. coli & 142 & $129(90.8)$ & $3(2.1)$ & $10(7.0)$ \\
Klebsiella spp & 21 & $14(66.7)$ & $7(33.3)$ & $0(0)$ \\
Enterococcus spp & 12 & $9(75.0)$ & $3(25.0)$ & $0(0)$ \\
Enterobacter spp & 10 & $5(50.0)$ & $3(30.0)$ & $2(20.0)$ \\
Pseudomonas spp & 10 & $9(90.0)$ & $1(10.0)$ & $0(0)$ \\
Other single organisms & 6 & $5(83.3)$ & $0(0)$ & $1(16.7)$ \\
Other identified & 15 & $7(46.7)$ & $5(33.3)$ & $3(20.0)$ \\
$\quad$ multiple organisms & & & & \\
\hline
\end{tabular}

Abbreviations: UTI, urinary tract infection. ${ }^{*}$ In vitro susceptibility of uropathogen to initial antibiotic. ${ }^{\dagger}$ In vitro nonsusceptibility of uropathogen to initial antibiotic. ${ }^{\ddagger}$ No antibiotics given on day of, or after, urine culture collection.

most common initial antibiotics were a third-generation cephalosporin $(39.1 \%)$, combination of ampicillin and a third- or fourth-generation cephalosporin $(16.7 \%)$, and combination of ampicillin with gentamicin $(11.1 \%)$. A third-generation cephalosporin was the initial antibiotic for $46.1 \%$ of the E. coli and $56.9 \%$ of Klebsiella spp UTIs. Resistance to third-generation cephalosporins but carbapenem susceptibility was noted for $4.5 \%$ of E. coli and $7.7 \%$ of Klebsiella spp isolates. Patients with UTIs caused by Klebsiella spp, mixed organisms, and Enterobacter spp were more likely to receive discordant antibiotic therapy. Patients with Enterobacter spp and mixed-organism UTIs were more likely to have delayed antibiotic therapy. Nineteen patients $(8.8 \%)$ had positive blood cultures. Fifteen $(6.9 \%)$ required intensive care unit (ICU) admission during hospitalization.

Unadjusted results are shown in Supporting Appendix 1 , in the online version of this article. In the adjusted analysis, discordant antibiotic therapy was associated with a significantly longer LOS, compared with concordant therapy for all UTIs and for all UTIs caused by a single organism (Table 3). In adjusted analysis, discordant therapy was also associated with a 3.1 day (IQR: 2.0, 4.7) longer length of stay compared with concordant therapy for all E. coli UTIs.

Time to fever resolution was analyzed for patients with a documented fever at presentation for each treatment subgroup. One hundred thirty-six patients were febrile at admission and 122 were febrile beyond the first recorded vital signs. Fever was present at admission in $60 \%$ of the concordant group and $55 \%$ of the discordant group $(P=0.6)$. The median duration of fever was 48 hours for the concordant group $(\mathrm{n}=107$; IQR: 24,240$)$ and 78 hours for the discordant group ( $\mathrm{n}=12$; IQR: 48, 132). All patients were afebrile at discharge. Differences in fever duration between treatment groups were not statistically significant $(P=0.7)$.
TABLE 3. Difference in LOS for Children With UTI Based on Empiric Antibiotic Therapy

\begin{tabular}{llc}
\hline Bacteria & Difference in LOS $(95 \% \mathrm{Cl})^{*}$ & $P$ Value \\
\hline All organisms & & \\
Concordant vs discordant & $-1.8(-2.1,-1.5)$ & $<0.0001$ \\
Concordant vs delayed antibiotics & $-1.4(-1.7,-1.1)$ & 0.01 \\
Single organisms & $-1.9(-2.4,-1.5)$ & $<0.0001$ \\
Concordant vs discordant & $-1.2(-1.6,1.2)$ & 0.37 \\
Concordant vs delayed antibiotics & \\
\hline
\end{tabular}

Abbreviations: $\mathrm{Cl}$, confidence interval; LOS, length of stay; UTI, urinary tract infection. ${ }^{*}$ Models adjusted for age, sex, race, presence of vesicoureteral reflux (VUR), chronic care condition, abnormal genitourinary (GU) anatomy, prophylactic antibiotic use.

\section{DISCUSSION}

Across 5 children's hospitals, 1 out of every 10 children hospitalized for UTI received discordant initial antibiotic therapy. Children receiving discordant antibiotic therapy had a 1.8 day longer LOS when compared with those on concordant therapy. However, there was no significant difference in time to fever resolution between the groups, suggesting that the increase in LOS was not explained by increased fever duration.

The overall rate of discordant therapy in this study is consistent with prior studies, as was the more common association of discordant therapy with non-E. coli UTIs. ${ }^{10}$ According to the Kids' Inpatient Database 2009 , there are 48,100 annual admissions for patients less than 20 years of age with a discharge diagnosis code of UTI in the United States. ${ }^{1}$ This suggests that nearly 4800 children with UTI could be affected by discordant therapy annually.

Children treated with discordant antibiotic therapy had a significantly longer LOS compared to those treated with concordant therapy. However, differences in time to fever resolution between the groups were not statistically significant. While resolution of fever may suggest clinical improvement and adequate empiric therapy, the lack of association with antibiotic concordance was not unexpected, since the relationship between fever resolution, clinical improvement, and LOS is complex and thus challenging to measure. ${ }^{21}$ These results support the notion that fever resolution alone may not be an adequate measure of clinical response.

It is possible that variability in discharge decisionmaking may contribute to increased length of stay. Some clinicians may delay a patient's discharge until complete resolution of symptoms or knowledge of susceptibilities, while others may discharge patients that are still febrile and/or still receiving empiric antibiotics. Evidence-based guidelines that address the appropriate time to discharge a patient with UTI are lacking. The American Academy of Pediatrics provides recommendations for use of parenteral antibiotics and hospital admission for patients with UTI, but does not address discharge decision-making or patient 
management in the setting of discordant antibiotic therapy. ${ }^{2,21}$

This study must be interpreted in the context of several limitations. First, our primary and secondary outcomes, LOS and fever duration, were surrogate measures for clinical response. We were not able to measure all clinical factors that may contribute to LOS, such as the patient's ability to tolerate oral fluids and antibiotics. Also, there may have been too few patients to detect a clinically important difference in fever duration between the concordant and discordant groups, especially for individual organisms. Although we did find a significant difference in LOS between patients treated with concordant compared with discordant therapy, there may be residual confounding from unobserved differences. This confounding, in conjunction with the small sample size, may cause us to underestimate the magnitude of the difference in LOS resulting from discordant therapy. Second, short-term outcomes such as ICU admission were not investigated in this study; however, the proportion of patients admitted to the ICU in our population was quite small, precluding its use as a meaningful outcome measure. Third, the potential benefits to patients who were not exposed to unnecessary antibiotics, or harm to those that were exposed, could not be measured. Finally, our study was obtained using data from 5 free-standing tertiary care pediatric facilities, thereby limiting its generalizability to other settings. Still, our rates of prophylactic antibiotic use, VUR, and GU abnormalities are similar to others reported in tertiary care children's hospitals, and we accounted for these covariates in our model..$^{22-25}$

As the frequency of infections caused by resistant bacteria increase, so will the number of patients receiving discordant antibiotics for UTI, compounding the challenge of empiric antimicrobial selection. Further research is needed to better understand how discordant initial antibiotic therapy contributes to LOS and whether it is associated with adverse short- and long-term clinical outcomes. Such research could also aid in weighing the risk of broader-spectrum prescribing on antimicrobial resistance patterns. While we identified an association between discordant initial antibiotic therapy and LOS, we were unable to determine the ideal empiric antibiotic therapy for patients hospitalized with UTI. Further investigation is needed to inform local and national practice guidelines for empiric antibiotic selection in patients with UTIs. This may also be an opportunity to decrease discordant empiric antibiotic selection, perhaps through use of antibiograms that stratify patients based on known factors, to lead to more specific initial therapy.

\section{CONCLUSIONS}

This study demonstrates that discordant antibiotic selection for UTI at admission is associated with longer hospital stay, but not fever duration. The full clinical consequences of discordant therapy, and the effects on length of stay, need to be better understood. Our findings, taken in combination with careful consideration of patient characteristics and prior history, may provide an opportunity to improve the hospital care for patients with UTIs.

Disclosure: Nothing to report.

\section{References}

1. HCUP Kids' Inpatient Database (KID). Healthcare Cost and Utilization Project (HCUP). Rockville, MD: Agency for Healthcare Research and Quality; 2006 and 2009. Available at: http:// www.hcup-us.ahrq.gov/kidoverview.jsp.

2. Subcommitee on Urinary Tract Infection, Steering Committee on Quality Improvement and Management. Urinary tract infection: clinical practice guideline for the diagnosis and management of the initial UTI in febrile infants and children 2 to 24 months. Pediatrics. 2011;128(3)595-610. doi: 10.1542/peds.2011-1330. Available at: http://pediatrics.aappublications.org/content/128/3/595. full.html.

3. Copp HL, Shapiro DJ, Hersh AL. National ambulatory antibiotic prescribing patterns for pediatric urinary tract infection, 1998-2007. Pediatrics. 2011;127(6):1027-1033.

4. Paschke AA, Zaoutis T, Conway PH, Xie D, Keren R. Previous antimicrobial exposure is associated with drug-resistant urinary tract infections in children. Pediatrics. 2010;125(4):664-672.

5. CDC. National Antimicrobial Resistance Monitoring System for Enteric Bacteria (NARMS): Human Isolates Final Report. Atlanta, GA: US Department of Health and Human Services, CDC; 2009.

6. Mohammad-Jafari H, Saffar MJ, Nemate I, Saffar H, Khalilian AR. Increasing antibiotic resistance among uropathogens isolated during years 2006-2009: impact on the empirical management. Int Braz J Urol. 2012;38(1):25-32.

7. Network ETS. 3rd Generation Cephalosporin-Resistant Escherichia coli. 2010. Available at: http://www.cddep.org/ResistanceMap/bugdrug/EC-CS. Accessed May 14, 2012.

8. Shaikh N, Ewing AL, Bhatnagar S, Hoberman A. Risk of renal scarring in children with a first urinary tract infection: a systematic review. Pediatrics. 2010;126(6):1084-1091.

9. Hoberman A, Wald ER. Treatment of urinary tract infections. Pediatr Infect Dis J. 1999;18(11):1020-1021.

10. Marcus N, Ashkenazi S, Yaari A, Samra Z, Livni G. Non-Escherichia coli versus Escherichia coli community-acquired urinary tract infections in children hospitalized in a tertiary center: relative frequency, risk factors, antimicrobial resistance and outcome. Pediatr Infect Dis J. 2005;24(7):581-585.

11. Ramos-Martinez A, Alonso-Moralejo R, Ortega-Mercader P, Sanchez-Romero I, Millan-Santos I, Romero-Pizarro Y. Prognosis of urinary tract infections with discordant antibiotic treatment [in Spanish]. Rev Clin Esp. 2010;210(11):545-549.

12. Velasco Arribas M, Rubio Cirilo L, Casas Martin A, et al. Appropriateness of empiric antibiotic therapy in urinary tract infection in emergency room [in Spanish]. Rev Clin Esp. 2010; 210(1):11-16.

13. Long SS, Pickering LK, Prober CG. Principles and Practice of Pediatric Infectious Diseases. 3rd ed. New York, NY: Churchill Livingstone/Elsevier; 2009.

14. National Committee for Clinical Laboratory Standards. Performance Standards for Antimicrobial Susceptibility Testing; Twelfth Informational Supplement.Vol M100-S12. Wayne, PA: NCCLS; 2002.

15. Tieder JS, Hall M, Auger KA, et al. Accuracy of administrative billing codes to detect urinary tract infection hospitalizations. Pediatrics. 2011;128(2):323-330.

16. Mongelluzzo J, Mohamad Z, Ten Have TR, Shah SS. Corticosteroids and mortality in children with bacterial meningitis. JAMA. 2008; 299(17):2048-2055.

17. Hoberman A, Wald ER, Penchansky L, Reynolds EA, Young S. Enhanced urinalysis as a screening test for urinary tract infection. Pediatrics. 1993;91(6):1196-1199.

18. Hoberman A, Wald ER, Reynolds EA, Penchansky L, Charron M. Pyuria and bacteriuria in urine specimens obtained by catheter from young children with fever. J Pediatr. 1994;124(4):513-519.

19. Zorc JJ, Levine DA, Platt SL, et al. Clinical and demographic factors associated with urinary tract infection in young febrile infants. Pediatrics. 2005;116(3):644-648.

20. Feudtner C, Hays RM, Haynes G, Geyer JR, Neff JM, Koepsell TD. Deaths attributed to pediatric complex chronic conditions: national trends and implications for supportive care services. Pediatrics. 2001; 107(6):E99. 
21. Committee on Quality Improvement. Subcommittee on Urinary Tract Infection. Practice parameter: the diagnosis, treatment, and evaluation of the initial urinary tract infection in febrile infants and young children. Pediatrics. 1999;103:843-852.

22. Fanos V, Cataldi L. Antibiotics or surgery for vesicoureteric reflux in children. Lancet. 2004;364(9446):1720-1722.

23. Chesney RW, Carpenter MA, Moxey-Mims M, et al. Randomized intervention for children with vesicoureteral reflux (RIVUR): back- ground commentary of RIVUR investigators. Pediatrics. 2008; 122(suppl 5):S233-S239.

24. Brady PW, Conway PH, Goudie A. Length of intravenous antibiotic therapy and treatment failure in infants with urinary tract infections. Pediatrics. 2010;126(2):196-203.

25. Hannula A, Venhola M, Renko M, Pokka T, Huttunen NP, Uhari M. Vesicoureteral reflux in children with suspected and proven urinary tract infection. Pediatr Nephrol. 2010;25(8):1463-1469. 